\title{
BMJ Open Do statins improve outcomes for patients with non-small cell lung cancer? A systematic review and meta- analysis protocol
}

\author{
Feng Li, ${ }^{1,2}$ Guangyu Liu, ${ }^{3}$ Raheleh Roudi, ${ }^{4}$ Qi Huang, ${ }^{5}$ Marc Swierzy, ${ }^{1}$ \\ Mahmoud Ismail, ${ }^{1}$ Song Zhao, ${ }^{2}$ Jens-Carsten Rueckert ${ }^{1}$
}

To cite: Li F, Liu G, Roudi R, et al. Do statins improve outcomes for patients with non-small cell lung cancer? A systematic review and metaanalysis protocol. BMJ Open 2018;8:e022161. doi:10.1136/ bmjopen-2018-022161

- Prepublication history and additional material for this paper are available online. To view these files, please visit the journal online (http://dx.doi. org/10.1136/bmjopen-2018022161).

$\mathrm{FL}, \mathrm{GL}$ and RR contributed equally.

Received 5 February 2018 Revised 20 July 2018 Accepted 6 August 2018
D) Check for updates

(c) Author(s) (or their employer(s)) 2018. Re-use permitted under CC BY-NC. No commercial re-use. See rights and permissions. Published by BMJ.

For numbered affiliations see end of article.

Correspondence to Professor Jens-Carsten Rueckert;

jens-c.rueckert@charite.de

\section{ABSTRACT}

Introduction Lung cancer is the most common neoplasm and the leading cause of cancer-related death worldwide. Non-small cell lung cancer (NSCLC), accounting for $85 \%$ of all lung cancer cases, is frequently diagnosed at an advanced and metastatic stage. In addition, survival of patients with NSCLC has not improved significantly over the recent decades. Statins are used as a cholesterollowering agent, but recently preclinical and clinical studies have revealed their anticancer effects. Thus, this systematic review and meta-analysis aims to clarify whether statins improve the prognosis of patients with NSCLC.

Methods and analysis We will search MEDLINE (PubMed), EMBASE, Web of Science, the Cochrane Central Register of Controlled Trials and ClinicalTrials.gov with no restriction on language. Both randomised controlled trials (RCTs) and observational cohort studies evaluating the prognostic role of statins in patients with NSCLC will be included. The primary outcome will be overall survival, and the secondary outcomes will include cancer-specific survival, disease-free survival and cancer recurrence. Two assessors will assess the RCTs using the Cochrane Collaboration's risk of bias tool and the observational cohort studies according to ROBINS-I. Publication bias will be assessed by funnel plot using the STATA software v.13.1.

Ethics and dissemination No ethical issues are predicted. This systematic review and meta-analysis aims to describe the prognostic effects of statins in patients with NSCLC, which would help clinicians to optimise treatment for patients with NSCLC. These findings will be published in a peer-reviewed journal and presented at national and international conferences.

PROSPERO registration number CRD42016047524.

\section{INTRODUCTION}

Lung cancer is the most common malignant tumour and the leading cause of cancer-related death all over the world. ${ }^{1}$ The incidence rate of lung cancer still shows an alarming trend. ${ }^{2}$ Clinically, small cell lung cancer and non-small cell lung cancer (NSCLC) are two main subtypes of lung cancer, of which
Strengths and limitations of this study

- This is the first systematic review aiming to summarise the evidence on whether statins improve the prognosis of patients with non-small cell lung cancer (NSCLC).

- Another strength could be the inclusion of both randomised studies and observational studies.

- This systematic review will be limited to excluding unpublished studies and including studies with the timing of intervention being both before and after the diagnosis of NSCLC.

NSCLC accounts for approximately $85 \%$ of the cases. ${ }^{134}$ In recent years, although studies have shown survival benefits from low-dose CT screening and tyrosine kinase inhibitors (TKI) specific for epidermal growth factor receptor (EGFR), ${ }^{5-11}$ the 5-year lung cancer-specific survival is only $18 \%{ }^{1}$ This is partly because most patients are diagnosed at an advanced stage of the disease, and a large proportion of patients with advanced NSCLC are resistant to radiotherapy and chemotherapy, as well as targeted therapy. ${ }^{14}$ Therefore, there is an urgent requirement to explore new therapies and optimise current treatment to improve the survival of patients with NSCLC.

Statins are inhibitors of 3-hydroxy 3-methylglutaryl-CoA (HMG-CoA) reductase, which is the rate-controlling enzyme in the production of mevalonate. ${ }^{12}$ Statins can reduce the plasma cholesterol level, and are the most commonly used drugs for preventing cardiovascular diseases and decreasing mortality and morbidity. ${ }^{13} 14$ Mounting evidence has demonstrated that statins are involved in the function of EGFR-related signalling pathways and c-Jun N-terminal kinase pathway. ${ }^{15}$ Furthermore, previous studies have indicated that statins exert anticancer effects by 


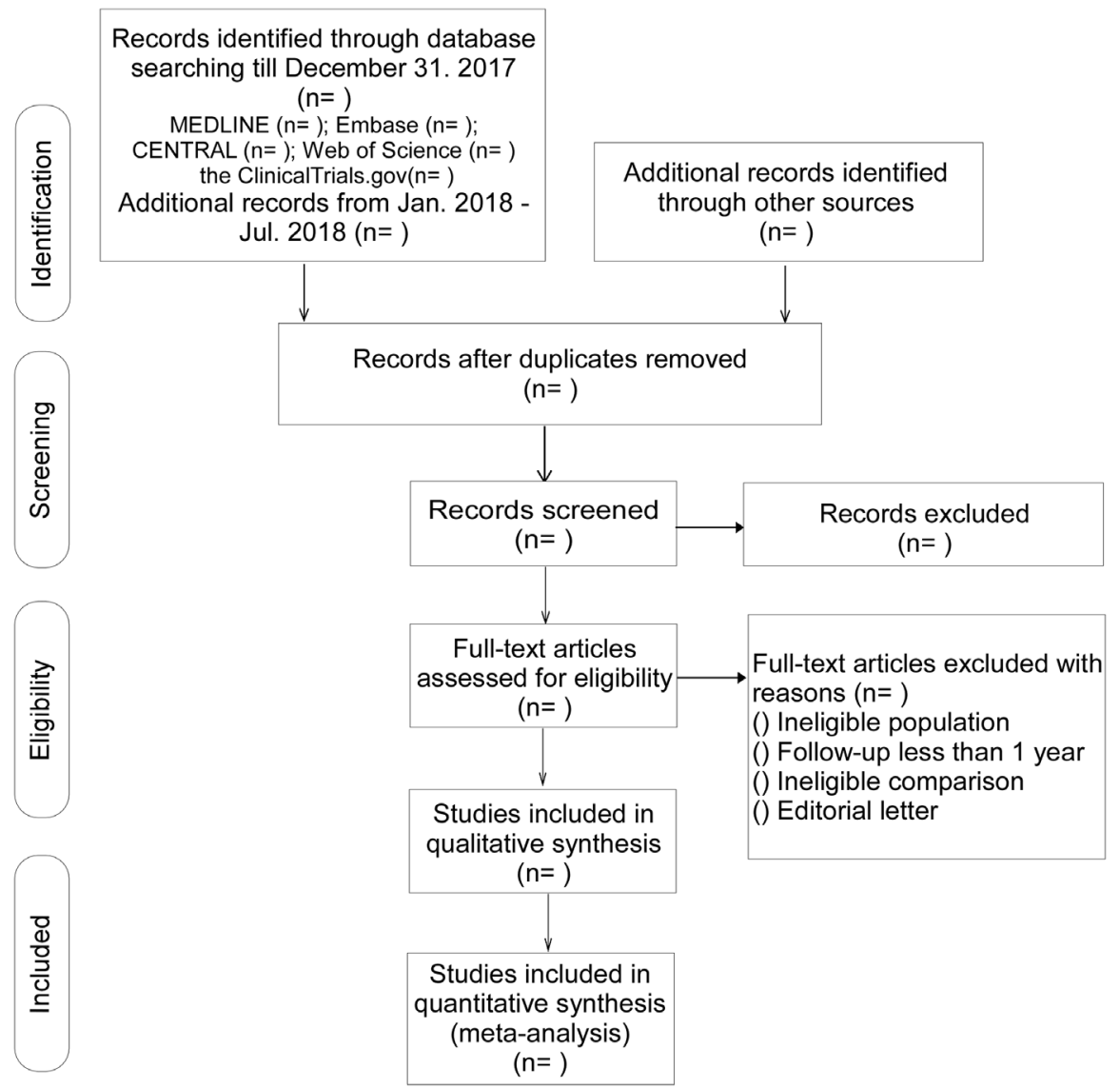

Figure 1 Flow chart showing identification and screening of eligible studies from MEDLINE, EMBASE, Cochrane Central Register of Controlled Trials (CENTRAL), Web of Science and the ClinicalTrials.gov for inclusion in this systematic review.

inducing apoptosis and inhibiting tumour cell growth and angiogenesis. ${ }^{16-18}$ Although it is controversial, many previous clinical publications suggest that statin use is associated with improved survival in patients with NSCLC. ${ }^{19-21}$ This systematic review and meta-analysis will explore the effects of statins in patients with NSCLC.

\section{OBJECTIVES}

This systematic review and meta-analysis aims to summarise the existing evidence to determine whether statins change the prognosis of patients with NSCLC. The primary objective is to demonstrate whether statins improve the overall survival of patients with NSCLC. The secondary objectives are to assess whether cancer recurrence, cancer-specific survival and disease-free survival differ between statins-using and non-statins-using patients with NSCLC.

\section{METHODS}

This systematic review has been developed in accordance with the guidelines detailed on the Preferred Reporting Items for Systematic Reviews and Meta-Analyses (PRISMA) checklist, ${ }^{22}$ and the protocol is reported according to its extension for protocols (PRISMA-P 2015). ${ }^{23} 24$ In addition, the flow chart (figure 1) will be employed in describing the study identification and selection process. The protocol of this systematic review has been registered in PROSPERO (trial registration number: CRD42016047524).

\section{INCLUSION CRITERIA \\ Type of studies}

We will include both randomised controlled trials (RCTs) and observational cohort studies. Studies assessing cell lines and animal models only, review articles, proceedings, conferences and case-control studies will be excluded.

\section{Type of participants}

We will include all patients of both genders who are diagnosed with NSCLC. Tumour classification will be made according to the 2011 WHO classification. ${ }^{25}$

\section{Type of intervention}

The intervention will be use of statins, which means patients who have taken statins at any type or dose before or after the diagnosis will be included in the treatment group.

\section{Type of control}

The control group will be patients who have been allocated to the control group during the study, which means 
the comparators given can be either placebos or no treatment of statins.

\section{Type of outcomes}

The primary outcome will be overall survival of patients with NSCLC. The secondary outcomes will include cancer recurrence, cancer-specific survival and diseasefree survival.

\section{SEARCH STRATEGY}

We will search the following databases: MEDLINE (PubMed), EMBASE, Web of Science, the Cochrane Central Register of Controlled Trials and the ClinicalTrials.gov, until 31 July 2018.

\section{PubMed search strategy}

The details of the PubMed database search strategy and syntax are sequentially provided in online supplementary appendix 1.

\section{Other resources}

The reference lists of the relevant original studies will be searched for additional studies, as well as reviews and key journals, such as 'Lung Cancer' (until 31 July 2018), 'Clinical Lung Cancer' (until 31 July 2018), 'Clinical Cancer Research' (until 31 July 2018), 'Journal of Clinical Oncology' (until 31 July 2018), 'JAMA' (until 31 July 2018), 'Lancet' (until 31 July 2018) and 'the New England Journal of Medicine' (until 31 July 2018). The hand-searching approach will be a manual page-by-page examination of all the issues.

There will be no restrictions on language or publication year. Studies written in languages other than English will be translated with the help of international scientists in our institutes. In addition, we will exclude unpublished studies.

\section{Data collection and analysis \\ Selection of studies}

Two reviewers will independently screen the titles and abstracts of all studies identified from the databases according to the inclusion criteria. Subsequently, the full texts of the identified studies will be reassessed independently by the two reviewers, verifying the reasons for inclusion and exclusion. Disagreements will be resolved by consulting a third reviewer.

\section{Data extraction}

A data extraction form will be developed, and the study data will be independently assessed and extracted by two reviewers. The following data will be extracted from all the included studies:

1. Study characteristics (author, year of publication, study design, setting, locations and patient enrolment strategies, and sample size).

2. Participants' characteristics (age, gender, ethnicity, smoking status, alcohol consumption, histopathological diagnosis, tumour, node, metastases (TNM) stage, history of treatment, associated comorbidities, timing of administration of statins, type of statins, dose of statins, comparator given and follow-up period).

If the reported data are not sufficient, we will contact the authors for further information. We will also pay extra attention to the confounding factors in the retrospective observational cohort studies.

Assessment of methodological quality

Two assessors will assess the methodological quality of RCTs using the Cochrane Collaboration's risk of bias tool $^{26}$ and observational studies according to The Risk Of Bias In Non-randomized Studies - of Interventions (ROBINS-I).${ }^{27}$ ROBINS-I is a new tool to assess risk of bias in non-randomised studies of interventions. ROBINS-I has seven domains of bias, including bias due to confounding, selection of participants, interventions themselves, deviations from intended interventions, missing data, measurement of outcomes and selection of the reported result. We have identified the following confounders: TNM stage and history of treatment (including chemotherapy, radiotherapy, targeted therapy and surgical therapy), which are important for the survival of patients with NSCLC. The reviewers should respond to the questions on signalling to judge the risk of bias in each domain, finally acquiring an overall risk of bias judgement for the outcome being assessed. Disagreements will be resolved by consulting a third independent reviewer.

\section{Measures of treatment effect}

The HR will be used to summarise survival data and dichotomous data, respectively, along with $95 \%$ CI. If an HR is not presented in an eligible study, for example only an OR or relative risk (RR) is available, we will estimate the HR using the information available in the study according to the methods reported in the previous studies. ${ }^{28}{ }^{29}$ Because if there are sufficient data to estimate an OR or RR, then there are usually sufficient data to estimate an HR. Also, if multiple effect estimates are presented in a paper, we will extract the result adjusted for the greatest number of our prespecified confounders. ${ }^{30}$

\section{Addressing missing data}

Missing relevant data will be excluded from analysis only after contacting the author but failing to acquire them. Sensitivity analysis will be conducted to explore if these missing data will influence the results of the meta-analysis.

\section{Assessment of heterogeneity}

Heterogeneity among studies will be measured using the Cochran $\mathrm{Q}$ test $\left(\chi^{2}\right)$ and $\mathrm{I}^{2}$ test. We will interpret it using the following guide: $\mathrm{I}^{2}, 0 \%-25 \%$ suggesting low heterogeneity, $25 \%-50 \%$ moderate heterogeneity and $75 \%-100 \%$ high heterogeneity.

\section{Assessment of reporting bias}

Since detecting and overcoming publication bias are problematic and firm guidance is not yet offered, we will 
use funnel plot to assess reporting bias, with results being interpreted cautiously.

\section{Strategy for data synthesis}

Extracted data will be entered into Review Manager V.5.3 software for aggregating risk estimates (HR and 95\% CI) by the first researcher and independently checked by the second one. We will use fixed-effect or random-effects methods as appropriate for analysis. If heterogeneity among the studies is identified as considerable, we will apply the random-effects method for analysis.

\section{Subgroup analyses}

Subgroup analyses are planned as follows:

1. Associated comorbidity such as hyperlipidaemia, coronary heart disease and so on.

2. Disease stage.

3. Cancer subtype.

4. Treatment type: surgery, chemotherapy and radiotherapy.

5. The time points at which patients start to take statins: prediagnosis or postdiagnosis.

6. Study type: RCT and retrospective studies.

7. Comparator given: placebo or no treatment.

Sensitivity analysis

A sensitivity analysis will be conducted to test the impact of the results with respect to the methodological quality items rated by the ROBINS-I and the Cochrane tool. Meta-analyses will be repeated after excluding studies with lower methodological quality and studies with high or unclear risk of bias. To evaluate the stability of the results, we will also conduct the leave-one-out sensitivity analysis. Sensitivity analyses will be reported with a summary table, and reviewed conclusions will be interpreted by making comparisons between the two meta-analyses.

\section{Confidence in cumulative evidence}

The quality of the evidence will be assessed using the scoring system of the Grading of Recommendations Assessment, Development and Evaluation Working Group. According to the final score, which will be acquired by summarising the score of each item in the scoring system, the quality of evidence is categorised into four levels: high ( $\geq 4$ points), moderate (3 points), low (2 points) and very low ( $\leq 1$ point). ${ }^{31}$

\section{Patient and public involvement}

Patients and the public were not involved in this study.

\section{DISCUSSION}

Lung cancer, especially NSCLC, is among the most common cancers for both men and women. Although many patients with NSCLC using EGFR-TKIs have achieved encouraging results, there are still a large proportion of patients who cannot benefit from them. Statins, considered as safe, cheap and effective drugs, are commonly used in the primary and secondary prevention of cardiovascular diseases; however, some preclinical and clinical studies have shed light on their anticancer effects and repurposed them as promising anticancer agents.

Our systematic review will clarify the prognostic effect of statins in patients with NSCLC, which would help patients and clinicians to optimise the treatment of NSCLC, especially patients with coexisting cardiovascular disease. This systematic review may also help guideline developers in the management of patients with NSCLC.

\section{ETHICS AND DISSEMINATION}

No ethical issues are predicted. This systematic review and meta-analysis will describe the prognostic effects of statins in patients with NSCLC, which would help clinicians to optimise the treatment for patients with NSCLC. These findings will be published in a peer-reviewed journal and presented at national and international conferences.

\section{Author affiliations}

${ }^{1}$ Department of General, Visceral, Vascular and Thoracic Surgery, Charite Universitatsmedizin Berlin, Berlin, Germany

${ }^{2}$ Department of Thoracic Surgery, The First Affiliated Hospital of Zhengzhou University, Henan, China

${ }^{3}$ Department of Anesthesiology, Peking University First Hospital, Beijing, China ${ }^{4}$ Department of Oncopathology, Research Center, Iran University of Medical Sciences, Tehran, Iran

${ }^{5}$ Department of Thoracic Surgery, Peking University People's Hospital, Beijing, China

Contributors FL and GL came up with the original idea of this work. QH assisted in protocol design. MS, MI, SZ and J-CR provided valuable advice for the research protocol. RR, FL and GL drafted the protocol, which was revised by all the authors. GL and FL will search for studies, extract and analyse the data, and QH will be consulted if they do not reach an agreement.

Funding The authors have not declared a specific grant for this research from any funding agency in the public, commercial or not-for-profit sectors.

Competing interests None declared.

Patient consent Not required.

Provenance and peer review Not commissioned; externally peer reviewed.

Open access This is an open access article distributed in accordance with the Creative Commons Attribution Non Commercial (CC BY-NC 4.0) license, which permits others to distribute, remix, adapt, build upon this work non-commercially, and license their derivative works on different terms, provided the original work is properly cited, appropriate credit is given, any changes made indicated, and the use is non-commercial. See: http://creativecommons.org/licenses/by-nc/4.0/.

\section{REFERENCES}

1. Siegel RL, Miller KD, Jemal A. Cancer statistics, 2017. CA Cancer J Clin 2017;67:7-30.

2. Chen W, Zheng R, Baade PD, et al. Cancer statistics in China, 2015. CA Cancer J Clin 2016;66:115-32.

3. Travis WD. Pathology of lung cancer. Clin Chest Med 2011;32:669-92.

4. Ettinger DS, Akerley W, Borghaei $\mathrm{H}$, et al. Non-small cell lung cancer. J Natl Compr Canc Netw 2012;10:1236-71.

5. Aberle DR, DeMello S, Berg CD, et al. Results of the two incidence screenings in the National Lung Screening Trial. N Engl J Med 2013;369:920-31.

6. Mok TS, Wu YL, Thongprasert S, et al. Gefitinib or carboplatinpaclitaxel in pulmonary adenocarcinoma. $N$ Engl $\mathrm{J}$ Med 2009;361:947-57. 
7. Maemondo M, Inoue A, Kobayashi K, et al. Gefitinib or chemotherapy for non-small-cell lung cancer with mutated EGFR.

N Engl J Med 2010;362:2380-8.

8. Zhou C, Wu YL, Chen G, et al. Erlotinib versus chemotherapy as first-line treatment for patients with advanced EGFR mutationpositive non-small-cell lung cancer (OPTIMAL, CTONG-0802): a multicentre, open-label, randomised, phase 3 study. Lancet Oncol 2011;12:735-42

9. Rosell R, Carcereny E, Gervais R, et al. Erlotinib versus standard chemotherapy as first-line treatment for European patients with advanced EGFR mutation-positive non-small-cell lung cancer (EURTAC): a multicentre, open-label, randomised phase 3 trial. Lancet Oncol 2012;13:239-46.

10. Sequist LV, Yang JC, Yamamoto N, et al. Phase III study of afatinib or cisplatin plus pemetrexed in patients with metastatic lung adenocarcinoma with EGFR mutations. J Clin Oncol 2013;31:3327-34.

11. Mendelsohn J, Baselga J. The EGF receptor family as targets for cancer therapy. Oncogene 2000;19:6550-65.

12. Tobert JA. Lovastatin and beyond: the history of the HMG-CoA reductase inhibitors. Nat Rev Drug Discov 2003;2:517-26.

13. Mihaylova B, Emberson J, Blackwell L, et al. The effects of lowering LDL cholesterol with statin therapy in people at low risk of vascular disease: meta-analysis of individual data from 27 randomised trials. Lancet 2012;380:581-90.

14. Mills EJ, Rachlis B, Wu P, et al. Primary prevention of cardiovascular mortality and events with statin treatments: a network metaanalysis involving more than 65,000 patients. J Am Coll Cardiol 2008:52:1769-81.

15. Osmak M. Statins and cancer: current and future prospects. Cancer Lett 2012;324:1-12.

16. Vallianou NG, Kostantinou A, Kougias M, et al. Statins and cancer. Anticancer Agents Med Chem 2014;14:706-12.

17. Mantha AJ, Hanson JE, Goss G, et al. Targeting the mevalonate pathway inhibits the function of the epidermal growth factor receptor. Clin Cancer Res 2005;11:2398-407.

18. Dimitroulakos J, Lorimer IA, Goss G. Strategies to enhance epidermal growth factor inhibition: targeting the mevalonate pathway. Clin Cancer Res 2006;12:4426s-31.
19. Lin JJ, Ezer N, Sigel K, et al. The effect of statins on survival in patients with stage IV lung cancer. Lung Cancer 2016;99:137-42.

20. Fiala O, Pesek M, Finek J, et al. Statins augment efficacy of EGFRTKIs in patients with advanced-stage non-small cell lung cancer harbouring KRAS mutation. Tumour Biol 2015;36:5801-5.

21. Han JY, Lee SH, Yoo NJ, et al. A randomized phase II study of gefitinib plus simvastatin versus gefitinib alone in previously treated patients with advanced non-small cell lung cancer. Clin Cancer Res 2011;17:1553-60.

22. Moher D, Liberati A, Tetzlaff J, et al. Preferred reporting items for systematic reviews and meta-analyses: the PRISMA statement. BMJ 2009;339:b2535.

23. Moher D, Shamseer L, Clarke M, et al. Preferred reporting items for systematic review and meta-analysis protocols (PRISMA-P) 2015 statement. Syst Rev 2015;4:1.

24. Shamseer L, Moher D, Clarke M, et al. Preferred reporting items for systematic review and meta-analysis protocols (PRISMA-P) 2015: elaboration and explanation. BMJ 2015;350:g7647.

25. Brambilla E, Travis WD, Colby TV, et al. The new world health organization classification of lung tumours. Eur Respir $J$ 2001:18:1059-68.

26. Higgins JP, Altman DG, Gøtzsche PC, et al. The cochrane collaboration's tool for assessing risk of bias in randomised trials. BMJ 2011;343:d5928.

27. Sterne JA, Hernán MA, Reeves BC, et al. ROBINS-I: a tool for assessing risk of bias in non-randomised studies of interventions. BMJ 2016;355:i4919.

28. Tierney JF, Stewart LA, Ghersi D, et al. Practical methods for incorporating summary time-to-event data into meta-analysis. Trials 2007;8:16.

29. Parmar MK, Torri V, Stewart L. Extracting summary statistics to perform meta-analyses of the published literature for survival endpoints. Stat Med 1998;17:2815-34.

30. Hunink MG, Wong JB. Meta-analysis of failure-time data with adjustment for covariates. Med Decis Making 1994;14:59-70.

31. Guyatt G, Oxman AD, Akl EA, et al. GRADE guidelines: 1. Introduction-GRADE evidence profiles and summary of findings tables. J Clin Epidemiol 2011;64:383-94. 